\title{
Hard Diffraction and Unitarity
}

\author{
V.A. Petrov \\ Institute for High Energy Physics, Protvino, 142280 Russia
}

- Unitarity of the $S$-matrix which stems from the postulate of asymptotic completeness (see e.g. [1]) refers to asymptotic states, representing physical particles. In quantum field-theoretic terms it means that one deals with on-shell, truncated Green functions. Unitarity is tightly related to (but not exhausted by) probabilistic interpretation of the scattering and production amplitudes, and effectively prevents these amplitudes from too fast growth with energy [2].

Hard processes in general, and hard diffraction in particular, are often related to off-shell amplitudes. Can unitarity, seemingly on-shell property, lead to limitations in this case also? In fact, unitarity of the $S$-matrix, when considered in the axiomatic framework, is assumed to hold off mass shell [3], thereof, e.g., the optical theorem holds when "external" particles are virtual.

However the bounds which were proven for the on-shell case cannot be derived for more general off-shell case.

This leads, in particular, to a possibility of a much faster rise with energy than in the on-shell case [4].

- In this talk we limit ourselves by consideration of deeply inelastic scattering (DIS) at small $x$, when it is believed to have mostly diffractive character.

In fact the most characteristic feature of a diffractive process is a diffractive pattern of the scattered waves. In high-energy collisions there is a related feature, i.e. a rapidity gap between diffractively scattered (excited, produced) final states. Nonetheless only the study of the diffractive pattern can give us as information about global properties (size, 
shape) of the scatterer ("interaction region"). From intuitive considerations one can think that for off-shell scattering the interaction radius should decrease with growth of virtuality.

- What is the role of unitarity? When asking such a question we mean the following. If one takes some "bare" or "Born" amplitude which is deduced from some simple arguments (say, Regge pole) it often violates unitarity or its consequences (e.g. Froissart-Martin (FM) bound). This is not the reason to abandon such a "wrong" amplitude which is considered to be very good in many other respects. The remedy is "unitarization", i.e. some infinite summation of the "bare" amplitude which yields a new, good amplitude respecting unitarity etc. The most known examples are eikonal and U-matrix representations.

Discovery of the fast growth of DIS cross-sections at HERA exacerbated the quest of possible unitarity-driven upper bounds.

Such bounds were obtained (see e.g.[5]) but at a price, after making serious extra assumptions which deprive the results of rigour and generality of the FM theorem.

- However the framework of general principles of quantum field theory seem to fairly admit power-like growth of the "off-shell" cross-sections. Extended Regge-eikonal just realizes this possibility in a concrete form. It is interesting to note that cross-sections of exclusive (binary) deeply virtual processes do not exceed the FM limit $\left(\log ^{2} s\right)$, while the corresponding total cross-sections grow as a power of energy [6]. It means that at extremely high $s$ and $Q^{2}$ "unitarity effects" for total crosssections are relatively negligible, while they are $100 \%$ important for binary exclusive cross-sections.

A natural interpretation of this phenomenon is that at high $Q^{2}$ the role of multiple production grows in full accordance whis ref [7].

We still lack the results concerning angular distribution of final particles in the binary deeply virtual exclusive processes. At the moment we can only mention the average impact parameter

$$
<b^{2}>\left(s, Q^{2}\right) \sim \log ^{2} s / \log Q^{2} .
$$

We see that the transverse interaction region grows asymptotically with 
energy (feature familiar from on-shell hadron-hadron processes) and shrinks with virtuality, $Q^{2}$, but slower.

At first sight it seems to mean that at equal c.m.s. energies the off-shell diffractive pattern is shallower and has more wide forward peak. But at realistic $s$ and $Q^{2}$ the picture can be much more complicated. We have to stress that up to now no sign of a dip is seen in the angular distribution of exclusively produced vector mesons at HERA. One could take this as an evidence in favour of a $Q^{2}$-induced spread of the diffractive pattern.

- The last subject I want to touch is the case when in capacity of a hard scale we take not the virtuality but the "compactification radius", $R_{c}$ ", assuming in accordance with newest ideas that our space-time has more than 4 dimensions, and that extra dimensions are somehow compactified. What is the rôle of a hard scale, $R_{c}$, in high energy behaviour? It appears that this rôle is quite insignificant. At least for the upper bound. One can show [8], that the compactification radius enters the upper bound (which is FM-like) quite harmfully, and, with a proper normalization, peacefully disappears in the zero limit bringing us back to the usual Minkowsky space-time and the FM bound. It is likely that influence of $R_{c}$ is more dramatic for high momentum transfers.

- As a conclusion I have no much to say.

1. Effects of fast growth of DIS cross-section discovered at HERA remain unexplained.

2. Unitarity does not limit this growth too stringently.

I express my deep gratitude to organizers of the magnificent workshop "Diffraction 2000" in Cetraro, especially to Roberto Fiore, Alessandro Papa, and Enrico Predazzi, for their kind hospitality and valuable support.

\section{References}

[1] N.N.Bogolyubov, A.A.Logunov, I.T.Todorov and A.I.Oksak. General Principles of Quantum Field Theory. Dordrecht: Kluwer, 1990. 
[2] M.Froissart, Phys. Rev. 123 (1961) 1053.

A.Martin, Phys. Rev. 129 (1963) 993.

[3] B.V.Medvedev, V.P.Pavlov, M.K.Polivanov and A.D.Sukhanov, TMF 13 (1972) 3.

[4] C.López and F.Yndurain, Phys. Rev. Lett. 44 (1980) 1118.

[5] S.M.Troshin and N.E.Tyurin, Preprint IHEP 96-29. Protvino, 1996;

A.L.Ayala, M.B.Gay Ducati and E.M.Levin, Phys.Lett. B388 (1996) 188.

[6] V.A.Petrov and A.V.Prokudin, Proceeding of the VIIIth Blois Workshop. (Eds. V.A.Petrov and A.V.Prokudin). World Scientific, p.95.

[7] A.V.Kisselev and V.A.Petrov, Preprint CERN TH-2000-075.

[8] V.A.Petrov, hep-ph/0008329. 